\title{
Maestros and bureaucrats
}

\section{Duncan Davies}

The Tender Ship: Governmental Management of Technological Change.

By Arthur M. Squires. Birkhauser: 1986. Pp.247. \$24.95.

Bureaucracies that disburse money selectively are feared and disliked by the recipients, even when the rules are clear and the process just. When, as in the funding of science and technology, the basis for choice is difficult or even impossible to codify, the dissatisfaction is permanent and acute. It is therefore a great achievement to write on this subject so as to fascinate, delight and inform the reader; $\mathrm{Dr}$ Squires has done just this. His secret is to tell a large number of case stories, ranging over centuries, using his broad perceptions as a teacher, a government contractor and helper, and a multidisciplinary researcher. So horribly and entertainingly does he expose the inadequacies of the present system, that radical reform seems essential.

Cleverly chosen material has been assembled by fourth-year students for seminars. In considerable detail, it shows how items as varied as a 17 th-century Swedish ship, the airship R101, a putative nuclear aeroplane, water desalination systems and an army rifle acquired unsuitable and idiotic design requirements and timetables as a result of personal ambition, fear, politicking, incompetence and place-seeking by remote officials. Contrasting and successful cases include the development of a gaseous diffusion plant to separate the uranium isotopes (in which project Squires was involved as a young worker), radar in Britain and the American wartime programme to develop synthetic rubber.

The key feature the successes share seems to be direction by "maestros of technology", who have large networks of equally competent friends to be brought in at critical moments. Another difference is that the successes began as essential wartime needs, based on clear decisions. The failures were peacetime projects lacking immediate and urgent need, which made them victims of entangled manoeuvring about design and priority. The nuclear aeroplane project, for example, oscillated between preference for direct heating of the working fluid, to indirect heating via a transfer fluid, so that a very doubtful starter never made headway. By contrast, those determining the course of the gas diffusion plant for uranium hexafluoride were "maestros of technology" motivated entirely by the urgent need to succeed. The one arbitrarily imposed change was to abandon flat membranes in favour of tubes. It was traumatic, and discarded those who were involved, and accelerated progress.

Squires believes in the mixed economy: the private sector for the product needed on a short-to-medium timespan for a price that definite customers will pay; and the public sector for the basic work, the longrange job and items that society needs but customers do not buy. It is interesting that research for the marketplace lies between his extremes. Target specifications for a vehicle, domestic gadget, telecommunications feature or food product are bound to change as the market alters, and become better understood as prototypes are tried out. Although such changes in aim are infuriating, expensive and disheartening, the market can be seen and understood better than a distant bureaucracy. It is known who is being captious or demanding, and to some extent, why. Utility, attractiveness and cost-effectiveness are not as incomprehensible and irrational as politicised fund-control. This adds point to the author's discontent about the funding methods used by governments in peacetime.

As might be expected from such an active and lively mind, the solution cautiously proposed is draconian and revolutionary. For the funding of basic science, Squires suggests replacing the incompetent and sometimes dishonest decision of the remote bureaucrat or committee by the judgement of applicants' previous research results rather than prospectuses, much work, but it made sense to all

and then paying 80 per cent of the prize money as block grants to their institution, with only 20 per cent going to the individual. Selection of the research projects would then be a local matter, and encourage laboratories to bid hard for the best people. Such an arresting idea is certainly bound to stimulate radical discussion.

The assessments for prize-awarding on this scale, however, would be very demanding on the time of able scientists, and it is hard to see that orthodoxy would not prevail. One could hardly find enough competent juries comprising people not doing research; peer-judgement seems inevitable, so that this week's judge will be next week's appellant. More thought seems to be needed, to avoid jumping from an uncomfortably hot frying pan into a red-hot fire.

Alongside the descriptions of work, there are perceptive analyses of the habits of bureaucracies, presidents, practitioners and professors, on whom Squires' distilled wisdom is well worth close attention. There are some mistakes; it seems unjust to say that the Solvay process for making alkali "quickly displaced the Leblanc process everywhere except in England" (p.119) when it was a British firm that built the first large Solvay plants and then exported them widely. However, with so much material so attractively assembled such errors are almost inevitable. After the first reading, this is a book for the bedside, to be dipped into from time to time

Duncan Davies, 3 Broadlands Close, London N6 $4 A F$, UK, formerly Chief Scientist and Engineer, Department of Trade and Industry, and Research and Development General Manager of ICI, is Chairman of British Ceramics Research Association Ltd and President of the Society of Chemical Industry.

\section{Molecular patterns}

\section{Robin Carrell}

The New Genetics and Clinical Practice, 2nd Edn. By D.J. Weatherall. Oxford University Press:1986. Pp.206. Hbk $£ 24.95, \$ 15.95 ; p b k £ 8.95$.

THE CHANGE in human genetics from a descriptive to a molecular science has occurred so rapidly that the discipline has been left with an outdated terminology and outmoded concepts. The consequence is a need for a profound revision of the whole subject to give what has become known as the "new genetics". It is not surprising that the clearest exponents of this new genetics have approached it indirectly, not as traditional geneticists. but laterally through the molecular studies of individual inherited diseases. This is the case with Professor Weatherall whose background has been the investigation of the disorders of haemoglobin synthesis.

The abnormal haemoglobin variants have long demonstrated that genetic diseases are usually syndromes with the same disease picture arising from a number of diverse mutations. Over 100 different mutations are known to give the characteristic anaemia of the unstable haemoglobins, of which nearly a third arise from new spontaneous mutations. This is the pattern now being seen in haemophilia where again a well-defined disease arises from a whole range of molecular variations, the occurrences of the disease being sustained by the continuous addition of new mutations. 